\title{
Dynamic Analysis of Hybrid DC-DC Converters
}

\author{
Alfonso Conesa, Herminio Martínez and José María Huerta \\ Technical University of Catalonia (EUETIB / UPC) \\ C/ Comte d'Urgell, 187. E-08036. Barcelona, SPAIN \\ E-Mail: \{alfonso.conesa, herminio.martinez\}@upc.edu
}

\section{Acknowledgements}

This work has been partially funded by project TEC2004-05608-C02-01 (-02) from the Spanish MCYT and EU FEDER funds.

\section{Keywords}

Converter circuit, Converter control, Voltage Regulator Modules, DC power supply, Modeling.

\begin{abstract}
This paper shows the analysis and implementation of a hybrid DC-DC power converter. The proposed topology consists of a series linear voltage regulator in parallel with a switching step-down converter. This topology can provide small ripple at the output voltage, fast responses for load variations and high efficiency for high load current conditions. In the hybrid structures there is not a classical feedback loop as in DC-DC converters, but they are feedback systems too. Therefore, their smallsignal analysis is important to assure stability of the implemented power supply system. From the analysis proposed we deduce the critical components that induce instability to the converter and how to improve the final design.
\end{abstract}

\section{I.- Introduction}

Series linear regulators have been structures widely used in power supply systems in applications of low or moderate currents and consume $[1,2,3]$. This kind of voltage regulators has several advantages that lead their use. However, in spite of these advantages, linear regulators present some serious drawbacks. For example, the efficiency of these structures hardly exceeds the $50 \%$ and the series-pass transistor has to hold up all the current demanded by the load. Thus, this component has to be dimensioned (both electrically and thermically) to dissipate an important quantity of energy, increasing the price, volume and weight in the supply system.

The drawbacks above presented are actually improved with the DC-DC switching converters $[4,5,6]$. The efficiency of these converters, in spite of not arriving at the $100 \%$ due to the omnipresent circuit losses, is near to this optimal value. But the design and implementation of this sort of converters is a more complex process than in linear regulators, especially their control loops when both line and load regulations are desired. In addition, the intrinsic switched nature of these converters leads to produce significant ripples in the output voltage and an increment of the EMIs in neighboring electronic systems.

In this article, a linear-switching hybrid converter (or linear-assisted converter) is implemented and modeled. These structures make good use of the advantages from previous alternatives (linear regulators and switching converters). Some of the aforementioned drawbacks are minimized as, for instance, the low efficiency and the high power dissipation in linear regulators, or the complexity in the design of the control for switching converters. Nevertheless, these structures have an intrinsic control loop. This loop maintains a constant output voltage and determinates the duty ratio of the switching converter, and could induce instabilities. The stability of the system depends on the values of the circuit components and the values of the load, in a similar way as linear regulators. Therefore, a 
stability analysis is mandatory in order to obtain design guidelines for this kind of DC-DC converters and assure its stability.

Figure 1 shows the simplest configuration. The voltage linear regulator guarantees the output voltage and the analog comparator $\left(C M P_{1}\right)$ controls the conduction or cut of the transistor $Q_{1}$, and fixes the switching frequency. Note that the objective of the switching converter is to provide the excess of current that the linear regulator does not supply, as equation (1) indicates.

$$
I_{\text {out }}=i_{\text {reg }}(t)+i_{L}(t)
$$

In a first approximation just do not consider hysteresis in the comparator $C M P_{1}$. The sense of the linear current $\left(i_{\text {reg }}\right)$ is done by means of $R_{\text {lim }}$ (shunt resistor). We can establish a boundary current value for the linear converter as (2).

$$
I_{\gamma}=\frac{V_{r e f}}{R_{\lim }}
$$

When $i_{\text {reg }}$ tends to increase $I_{\gamma}$ the $Q_{1}$ switch is $\mathrm{ON}$, and when $i_{\text {reg }}$ tends to decrease $I_{\gamma}$ the switch $Q_{1}$ is OFF. The current $I_{\text {out }}$ is a constant value, and $i_{L}$ increases or decreases in linear form. Consequently, the current sharing holds equation (1) as we can see in the waveforms in figure 2.

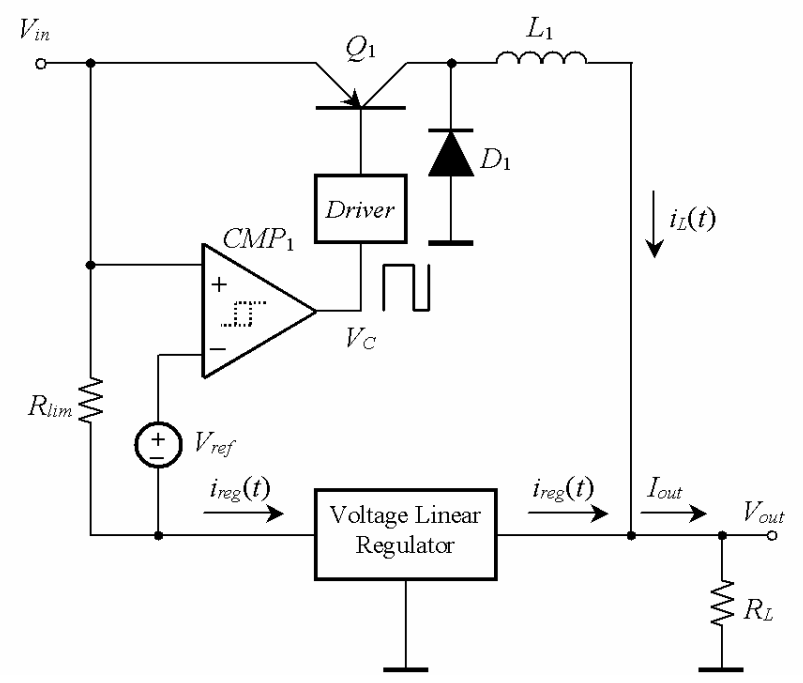

Fig. 1: Basic structure of a self-switched hybrid regulator.

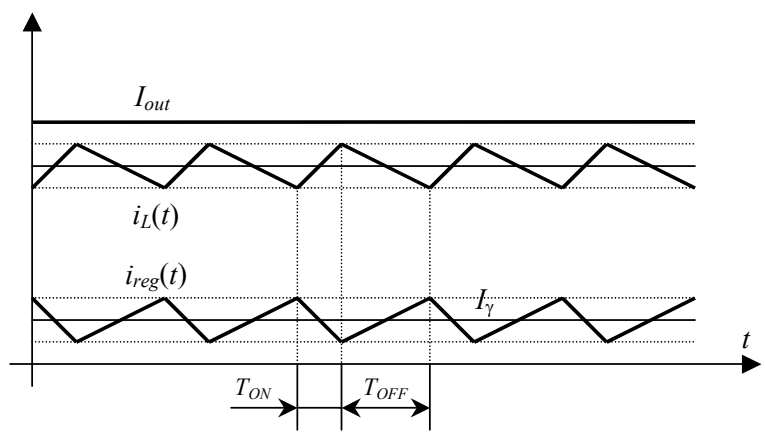

Fig. 2: Currents through the load resistor $\left(I_{\text {out }}\right)$, inductance $L_{1}\left(i_{L}\right)$ and linear regulator $\left(i_{\text {reg }}\right)$ in the steady-state.

In practical implementation of the converter, the current $I_{\gamma}$ should be the minimum and necessary value in order to make the linear regulator work properly, without penalizing its good regulation characteristics, and keep its losses bounded. In addition, the comparator $C M P_{1}$ must include a 
hysteresis to limit the maximum value of the switching frequency and, thus, the switching losses of the converter. The switching frequency of the converter is given by:

$$
f_{S}=\frac{R_{\text {lim }}}{L_{1}} \frac{R_{1}+R_{2}}{R_{1} V_{\text {sat }}} V_{\text {out }}\left(1-\frac{V_{\text {out }}}{V_{\text {in }}}\right)
$$

being $V_{\text {sat }}$ the positive saturation voltage at the output of the comparator, close to $V_{C C}$. The resistors $R_{1}$ and $R_{2}$ of the Schmitt trigger establish the upper and lower switching threshold levels of the comparator.

\section{II.- Implementation of the Hybrid Converter}

Figure 3 shows the practical implementation of the self-switched hybrid converter. The reference $\mathrm{D}_{2}$ fixes the voltage $V_{\text {ref }}$ that limits the maximum current through the linear regulator. The linear block is implemented with $O A_{2}, Q_{2}$, and resistors $R_{5}$ and $R_{6}$. The precision voltage reference $D_{3}$ (or zener diode) determines the output voltage of the whole circuit. The current $I_{\gamma}$ has been adjusted in this case, according to expression (2), to $50 \mathrm{~mA}$.

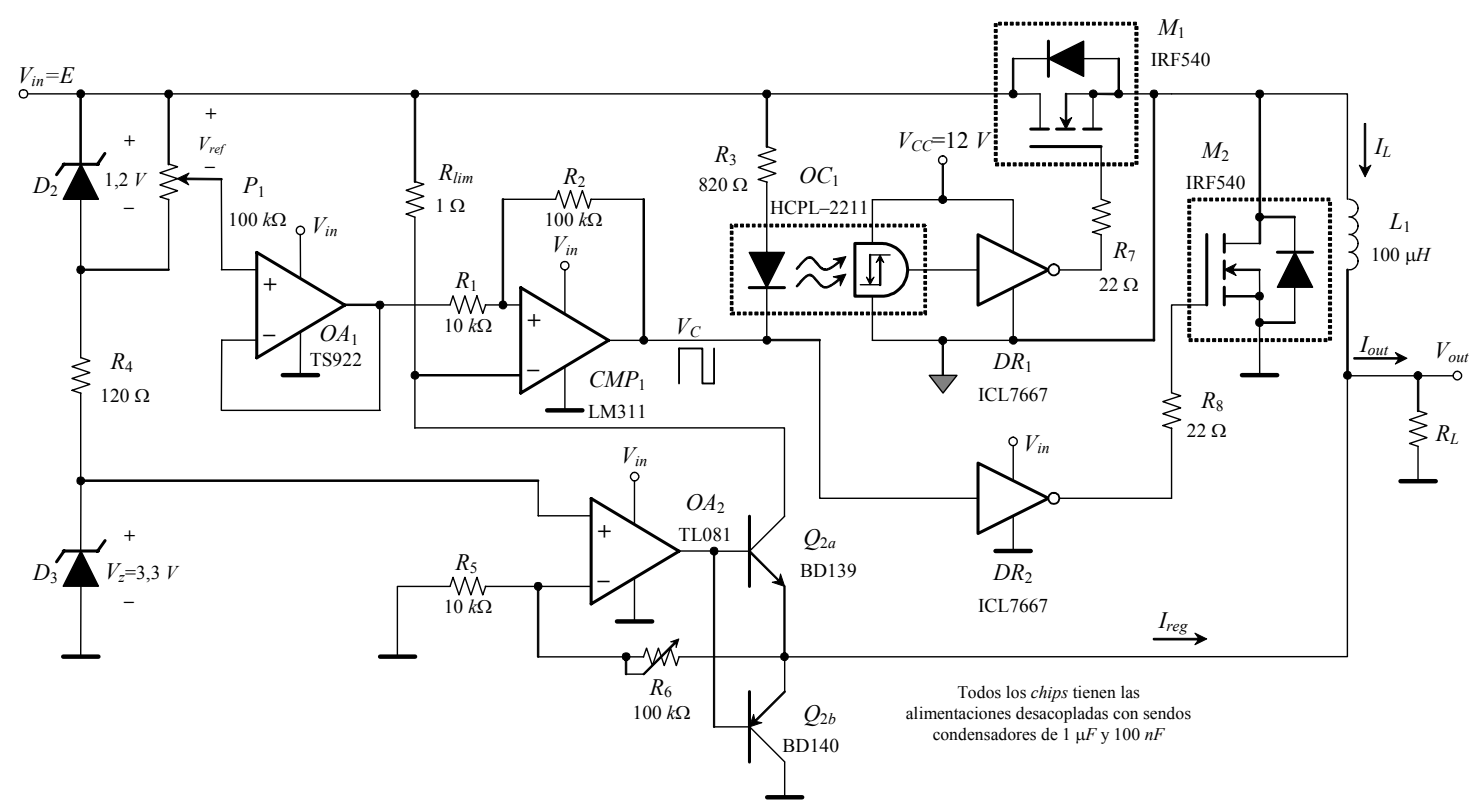

Fig. 3: Schematic of the hybrid converter prototype used to validate the proposed structure.

Figure 4 shows the simulation of a transient of the converter with $V_{i n}=E=10 \mathrm{~V}$. It is observed the response of the system to an input voltage step from $10 \mathrm{~V}$ to $13 \mathrm{~V}$ in time instant $t=20 \mu \mathrm{s}$, and a step change of the load resistor of the $50 \%$, from $10 \Omega$ to $5 \Omega$ in $t=40 \mu \mathrm{s}$. As we can see, the converter presents an excellent response.

Figure 5 shows the experimental waveforms of the output voltage $V_{\text {out }}$ (channel 1) and the cathode voltage of the diode $D_{1}$ (channel 2) for the hybrid regulator with $V_{Z}=3.3 \mathrm{~V}$ and an output current equal to $1 \mathrm{~A}$.

Figure 6 shows the efficiency achieved in the hybrid regulator as a function of the load current, with a margin from $0 \mathrm{~A}$ to $5 \mathrm{~A}$. Measures have been done for a threshold current $I_{\gamma}$ through the linear regulator of $50 \mathrm{~mA}$. In a wide margin of load conditions, the efficiency swells the $85 \%$ and is independent of the output current. In case of light load the efficiency decrease and tend to conventional linear regulator efficiency. 

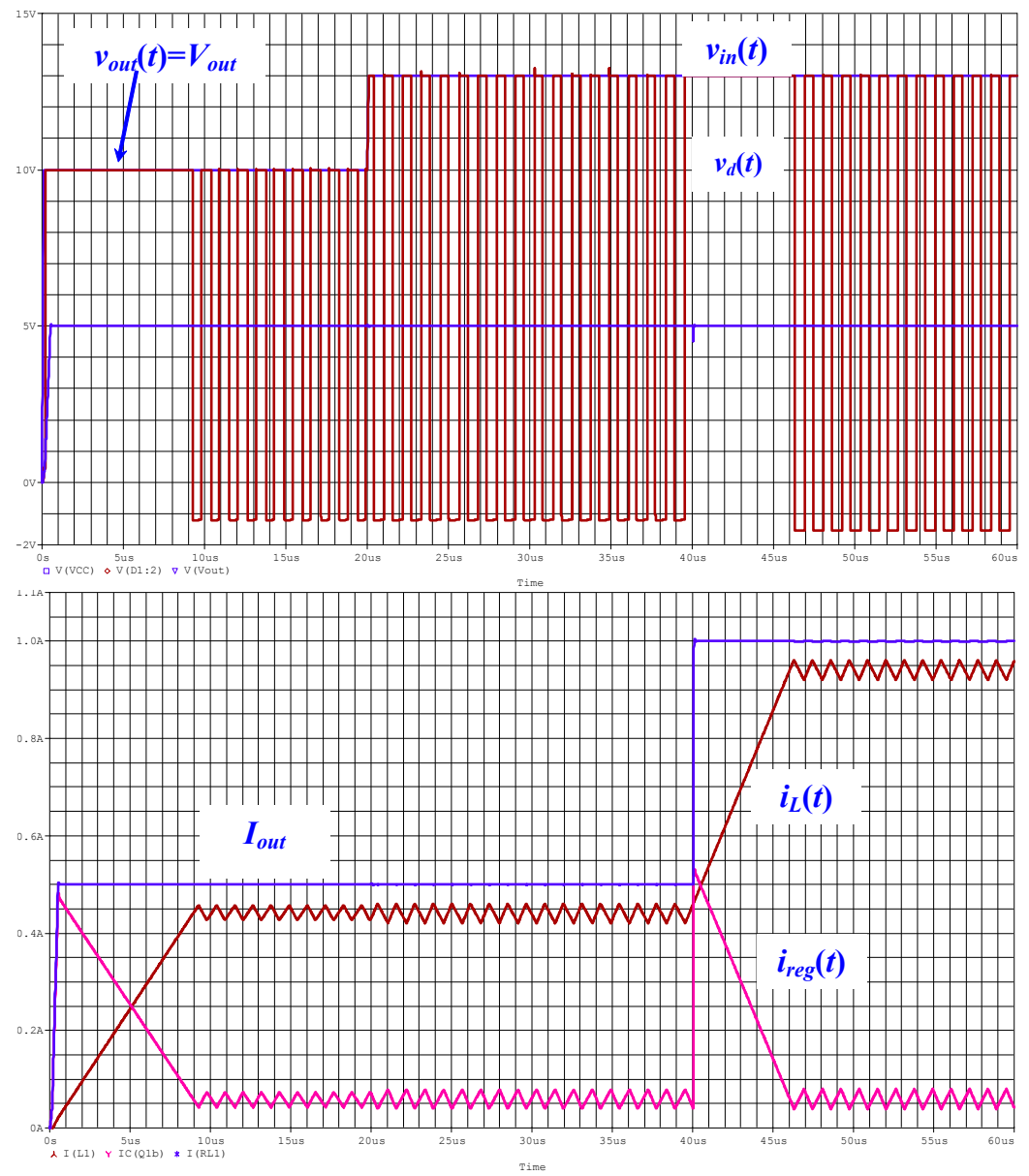

Fig. 4: Transient response of the hybrid converter to input voltage and load perturbations.

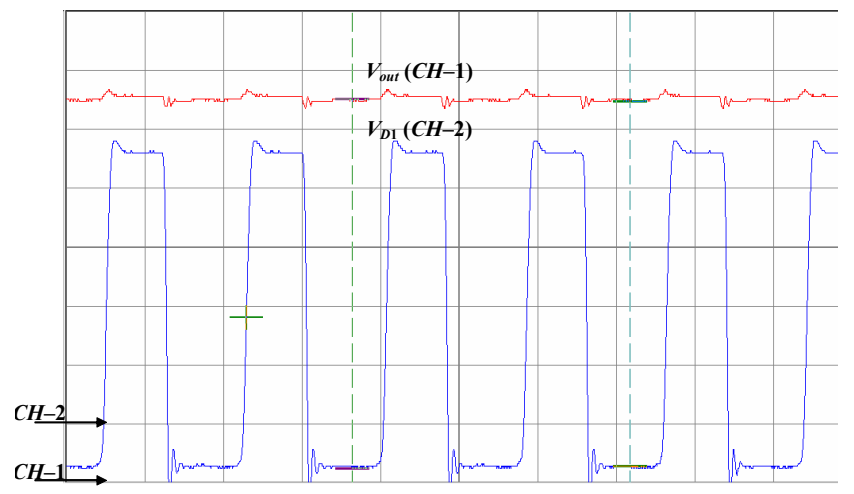

Fig. 5: Experimental waveforms of output voltage $V_{\text {out }}$ (channel 1) and cathode voltage of the diode $D_{1}$ (channel 2). Channel 1: 0,5 V/div. Channel 2: 1 V/div. TB: $1 \mu$ s/div.

\section{III.- Small signal analysis of the Hybrid Converter}

Linear-assisted converters, as we can see in figures 1 and 3, do not include the classical output capacitor. The linear regulator included in the hybrid converter guarantees the output voltage value and a free ripple waveform. But it is interesting to consider the effect of an output capacitor in the converter. This component can be added in order to eliminate possible transitory peaks in the output voltage, or must be considered when load nature is capacitive.

The presence of an output capacitor or parasitic capacitance load can induces a trend to instability in the converter. This instability is reflected as a considerable increment of the output-voltage ripple 
around the desired average output voltage and large fluctuations of the duty cycle of the switched converter. The switching frequency experiments large variations respect to the null output capacitance case and the frequency value not correspond to equation (3). In addition, the current $i_{\text {reg }}$ of the linear converter increases and the efficiency of the hybrid converter drops significantly.

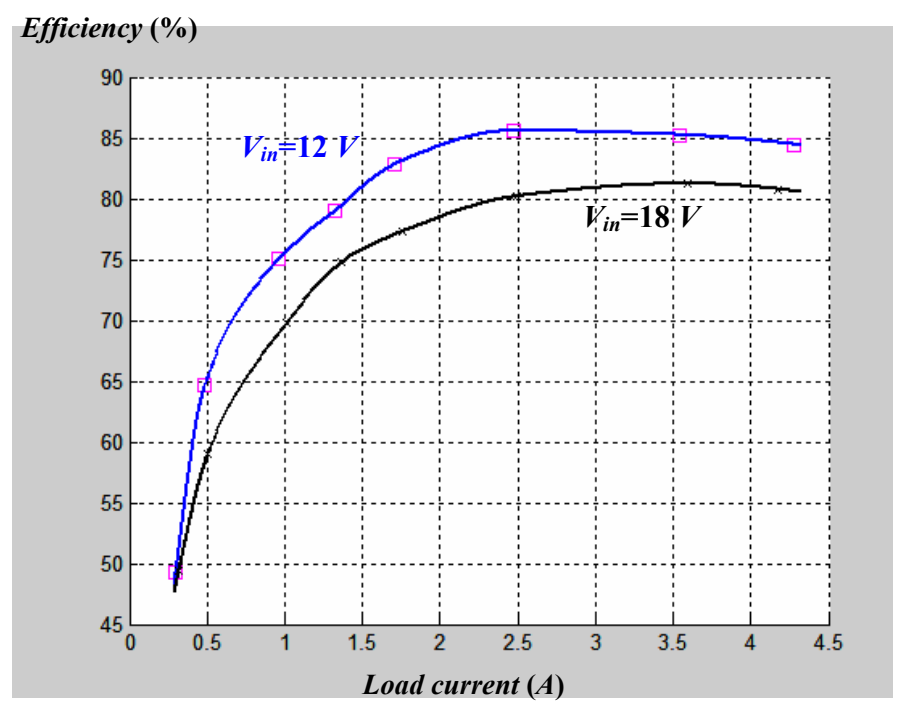

Fig. 6: Efficiency of the experimental hybrid converter versus the load current.
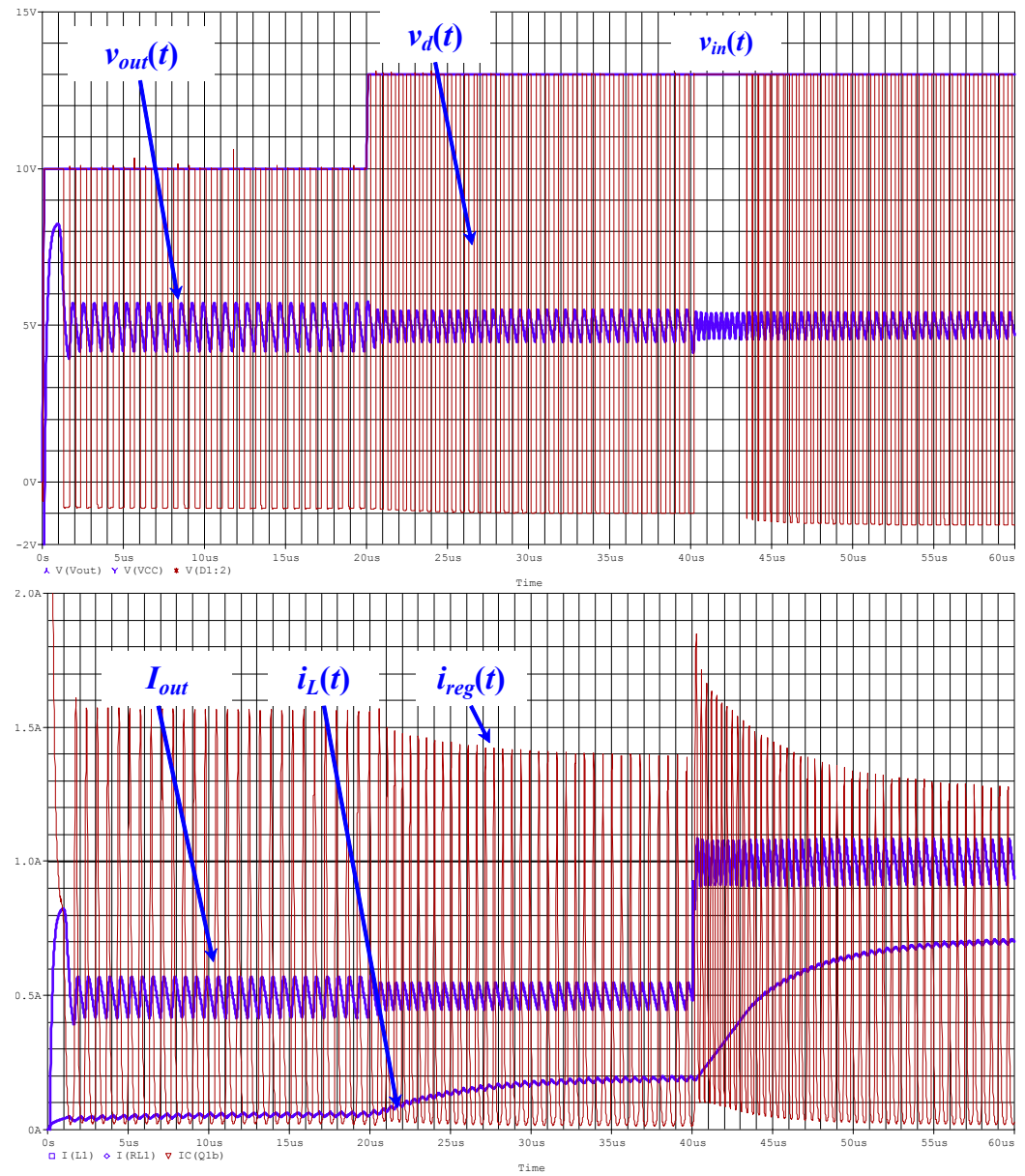

Fig. 7: Transient response of the linear-assisted converter with an output capacitance $C_{L}=100 n F$. 
Figure 7 shows the transient response of the linear-assisted converter in the same conditions that figure 4 but with an output capacitance $C_{L}=100 \mathrm{nF}$ and an equivalent series resistance (ESR) equal to $10 \mathrm{~m} \Omega$. Note that the inclusion of this $C_{L}$ network makes different the current sharing considered in expression (1) and the ideal current waveforms presented in figure 2.

Therefore, the $C_{L}$ has to be an agreement value. This value must be high enough to help to cancel perturbations but guaranteeing stability in the supply system. The experimentation in the prototype determines that together with the output capacitance some others parameters affect the stability of the converter. The most important components are the equivalent series resistance of the output capacitance $C_{L}$, the load resistor $R_{L}$ and the inductor $L_{1}$.

All these points make necessary to analyze the converter in order to obtain design approaches that guarantee its stability. The model considered of the linear-assisted DC-DC converter is presented in figure 8.

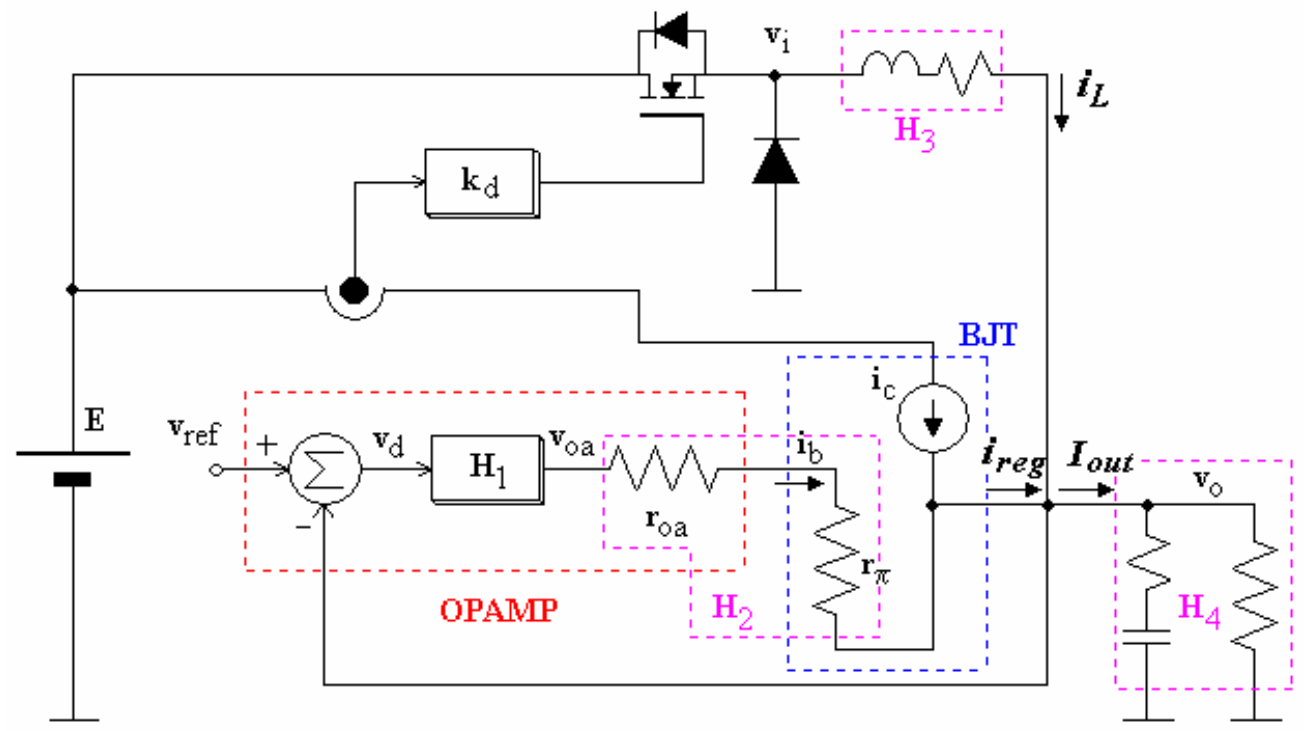

Fig. 8: Proposed model of the hybrid converter.

For the operational amplifier its low-pass response is considered in block $H_{1}$, with a differential gain $\left(A_{d}\right)$ and a dominant pole $\left(\omega_{o}\right)$, and its output resistance $\left(r_{o a}\right)$. For BJT transistor (working in its linear zone) the base-emitter dynamic resistance $\left(r_{\pi}\right)$ and a current gain $(\beta)$ are considered. Thus the block $H_{2}$ contains only the association of resistors $r_{o a}$ and $r_{\pi}$. The $r_{\pi}$ value is calculated as usual from equation (4):

$$
r_{\pi}=\frac{d v_{b e}}{d i_{b}}=\frac{\beta}{I_{C q}} \frac{v_{T}}{\lambda}
$$

The $k_{d}$ block makes sense of collector current in order to obtain the conducting ratio of switching transistor. Its value is given by equation (5).

$$
k_{d}=\frac{\Delta d}{\Delta i_{c}}=\frac{1}{i_{c, \max }}
$$

The inductor small signal voltage is $v_{i}$ and defined as usual as:

$$
v_{i}=(d \cdot E+e \cdot D)-v_{o}
$$


The inductor current $\left(i_{s}\right)$ is obtained thanks to the voltage $v_{i}$ and the block $H_{3}$, which models the inductor and its series resistance. Finally, with output current $\left(i_{o}\right)$ and block $H_{4}$, the output voltage $\left(v_{o}\right)$ is obtained. In the block $H_{4}$ the load resistance and output capacitor with its ESR is modeled.

In figure 9 the flow graph of the modeled linear-assisted converter is presented.

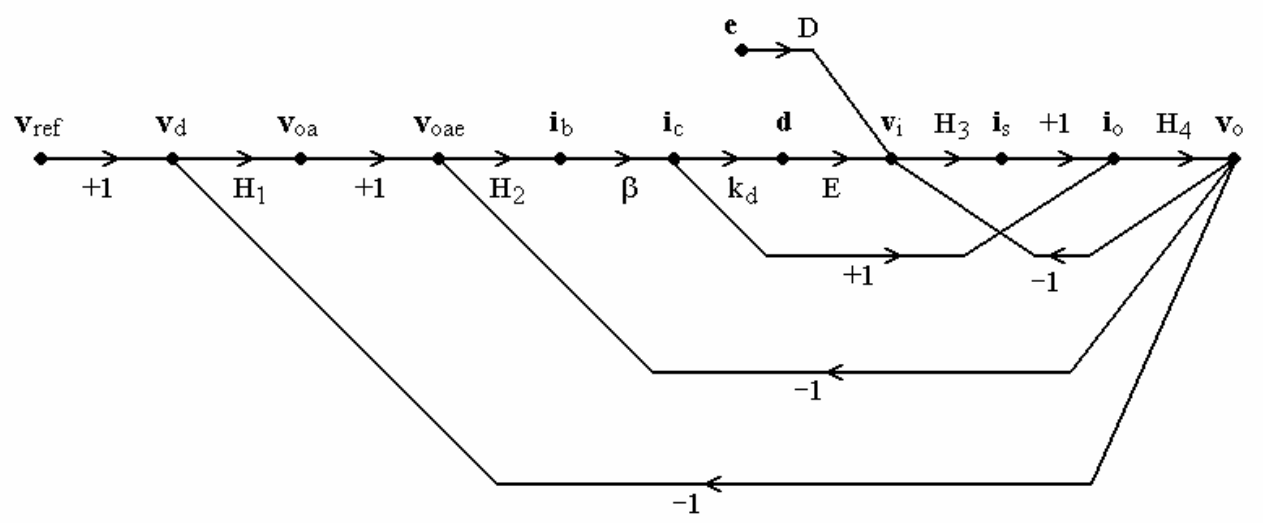

Fig. 9: Flow graph of the modeled linear-assisted converter.

Applying the Mason rule we obtain the transfer function given by:

$$
G(s)=\frac{v_{o}(s)}{v_{r e f}(s)}
$$

In Table I the Maple ${ }^{\circledR}$ code is presented in order to obtain the transfer function of equation (7). The result is simulated with Matlab ${ }^{\circledR}$ to obtain the poles-zeros map of the system (pzmap function). The used simulation values are close to the experimental values of the implemented prototype (Table II).

From the transfer function (7), and applying the Routh-Hurwitz criterion, it is possible obtain a design equation for the linear-assisted converter that determines its stability as a function of the circuit components. The denominator of the transfer function is a $3^{\text {rd }}$ order polynomial in the form

$$
a_{3} s^{3}+a_{2} s^{2}+a_{1} s+a_{0}
$$

The coefficients $a_{0}, a_{1}, a_{2}$ and $a_{3}$ of equation (8) consist of a considerable number of terms. The system will be stable if the condition (9) is satisfied, which involves that all the coefficients of the characteristic equation are positives.

$$
a_{1} a_{2}>a_{3} a_{0}
$$

Removing from the inequality (9) the negligible terms, reorganizing only the most important terms in the expression and isolating the capacitance $C_{L}$, it results:

$$
C_{L}<\frac{\left(\beta R_{L}+r_{d}+r_{o a}\right)\left(k_{d} E+A_{o a} \omega_{o a} L_{1}\right)}{A_{o a} \omega_{o a} k_{d} R_{L} E\left(r_{d}+r_{o a}\right)}
$$

For the design values considered in the prototype converter, the equation (10) can be simplified to equation (11)

$$
C_{L}<\frac{2 L_{1}}{k_{d} R_{L} E}
$$


because $\beta R_{L} \approx r_{d}+r_{o a}$ and $k_{d} E<<A_{o a} \omega_{o a} L_{1}$.

As experimental studies predicts, there are a strong influence in the stability of the converter with the inductor $L_{1}$, the load $R_{L}$, and the input voltage $E$ and the sense of the linear current $i_{\text {reg }}$ by means of $k_{d}$ parameter. From equation (10) the critical value of $C_{L}$ for the experimental components of the converter is $1 \mu \mathrm{F}$.

Table I: The Maple ${ }^{\circledR}$ code used to obtain the transfer function given in equation (6)

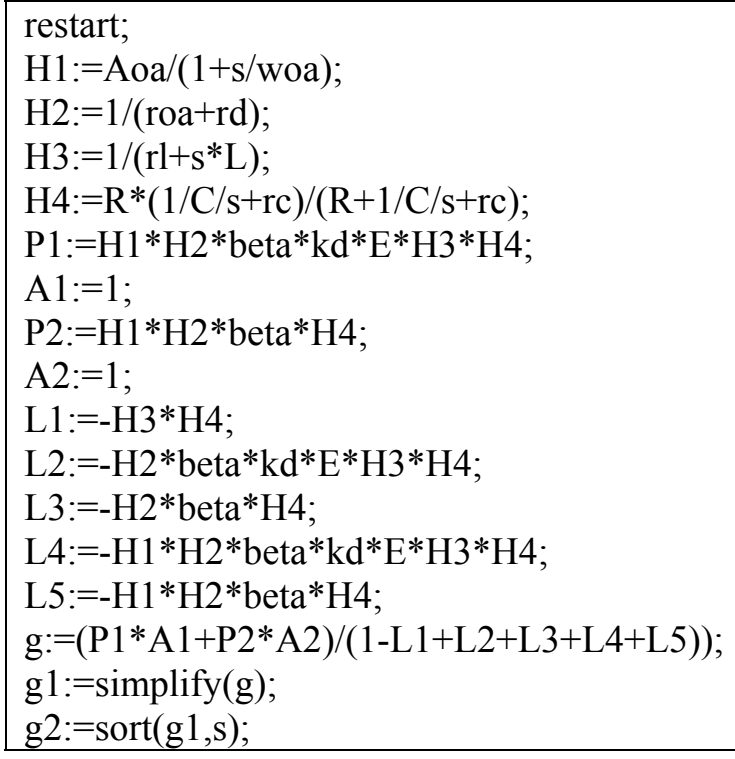

\section{IV.- Simulation and Experimental Results}

From the model proposed in previous section, some interesting simulations have been carried out in order to determine the stability of the system as a function of different circuit parameters.

Figure 10 presents the root locus diagram of the transfer function (7). The figure shows three set of curves for different values of the capacitor ESR $(1 \mathrm{~m} \Omega, 10 \mathrm{~m} \Omega$ and $100 \mathrm{~m} \Omega)$. In the three plots, the capacitor values goes from $C_{L}=100 \mathrm{nF}$ to $C_{L}=10^{4} \mu \mathrm{F}$. Note that when the ESR has a low value, the system tends easily to the instability when the capacitance value is higher than $1 \mu \mathrm{F}$. On the other hand, when the capacitor ESR is relatively high, the stability of the system is assured, even when output capacitance is high.

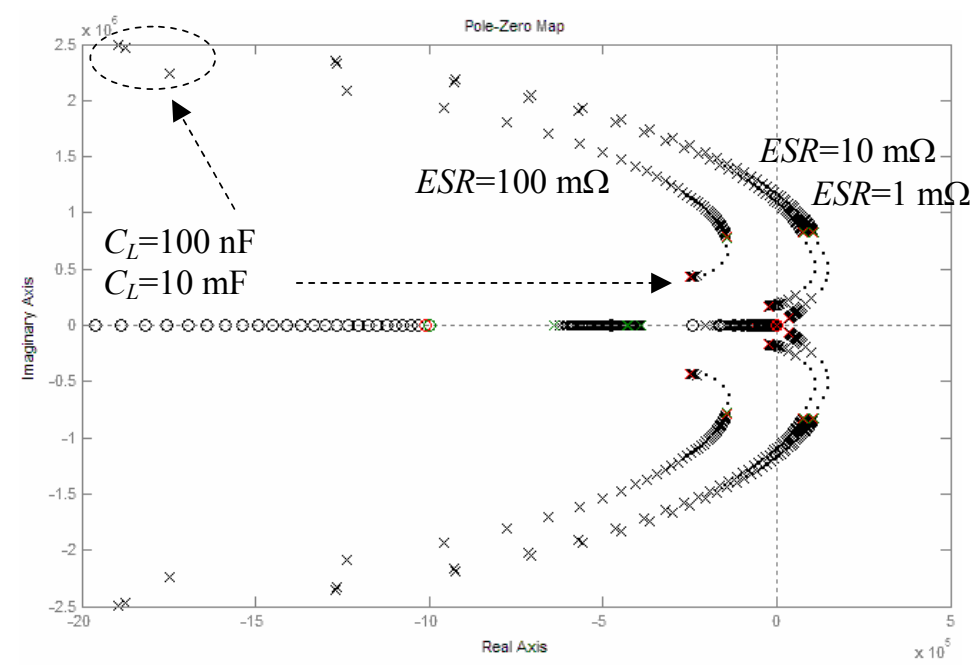

Fig. 10: Root locus diagram for different values of capacitor ESR and output capacitance $C_{L}$. 
Figure 11 shows again a root locus diagram of transfer function (7) for three different values of the inductor $L_{1}(200 \mu \mathrm{H}, 100 \mu \mathrm{H}$ and $50 \mu \mathrm{H})$. As previous figure, the three plots show the results for the capacitor values from $100 \mathrm{nF}$ to $10000 \mu \mathrm{F}$. When inductance $L_{1}$ has a small value, the converter tends to instability for small values of output capacitance.

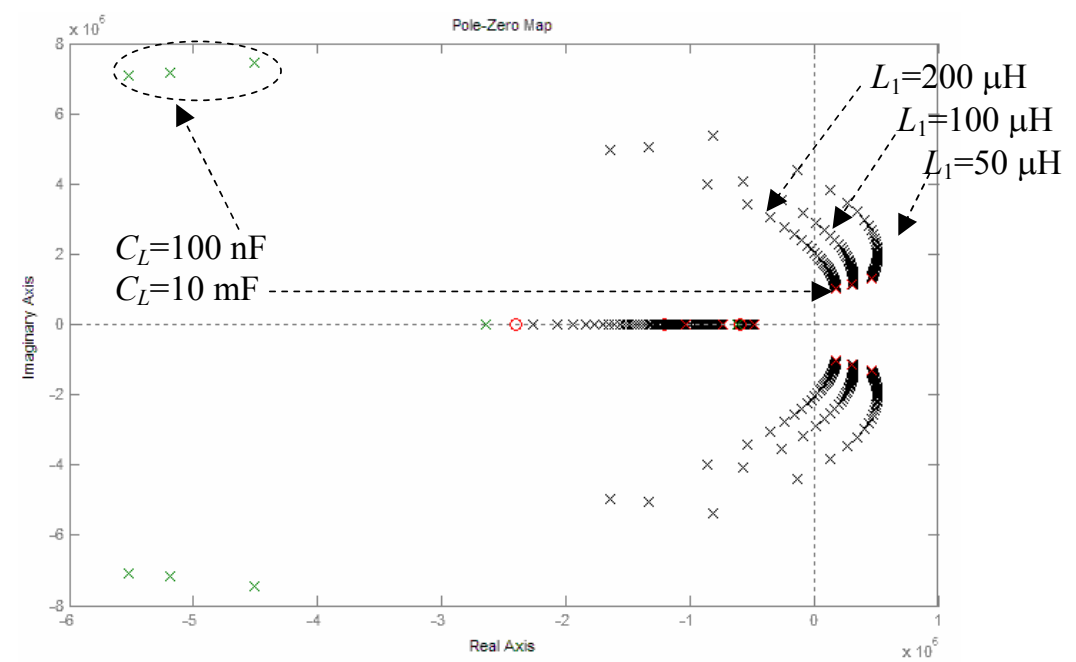

Fig. 11: Root locus diagram for different values of the inductor $L_{1}$ and output capacitance $C_{L}$.

Finally, figure 12 presents a root locus diagram of transfer function (7) for three different values of the load $R_{L}(0.2 \Omega, 2 \Omega$ and $20 \Omega)$. As previous figures, the capacitor values goes from $100 \mathrm{nF}$ to $10 \mathrm{mF}$. Note that when the load resistor has a high value, the system tends to instability when the capacitance value increases.

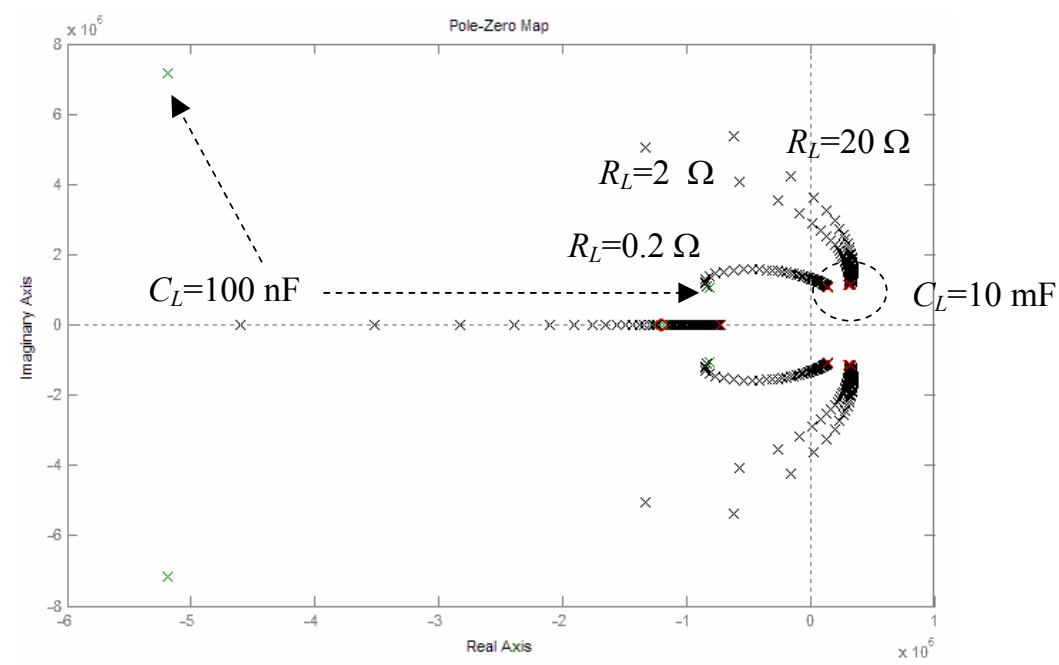

Fig. 12: Root locus diagram for different values of the load resistor $R_{L}$ and output capacitance $C_{L}$.

Varying different circuit parameters, other set of plots can be obtained in the same way that the obtained in previous figures. However, the aforementioned parameters $\left(C_{L}\right.$, capacitor ESR, $L_{l}$ and load resistance) are the four more important that determine the stability of the linear-assisted converter. Other parameters as, for instance, the inductor series resistance, the open loop gain of the op-amp, or the output resistance of the op-amp have an effect on the dynamics of the converter but actually do not lead the converter to the instability.

For the considered values given in Table II, the limit capacitor that assures the stability of the converter is equal to $1 \mu \mathrm{F}$. This value coincides with the experimental results obtained experimentally in the laboratory. 


\section{V.- Conclusions}

In this article, the implementation and subsequent modeling of a linear-assisted converter has been shown. The proposed converter is composed by a switching-linear hybrid structure. The system has a control loop to maintain a constant output voltage. This closed loop can induce instabilities as a function of the values of the circuit components and the load (in a similar way that linear regulators). Therefore, a stability study is mandatory in order to obtain design guidelines for this kind of DC-DC converters and assure the stability of the implemented power supply system. From the analysis we can establish criterion for selection of components and load conditions for the converter. The analysis makes evident that $C_{L}$, capacitor ESR, inductance $L_{1}$ and load resistance are the most influential circuital parameters on the stability of the hybrid converter. In addition, we remark the importance of the capacitor ESR value and nature of the load.

\section{VI.- References}

[1] R. J. Widlar: New Developments in IC Voltage Regulators. IEEE Journal of Solid-State Circuits, vol. SC-6 $\left(n^{\circ} 1\right)$ : pp. 2-7, February, 1971.

[2] R. K. Dokania, and G. A. Rincón-Mora: Cancellation of Load Regulation in Low Drop-Out Regulators. Electronic Letters, vol. 38 ( $\mathrm{n}^{\circ}$ 22): pp. 1300-1302, 24th October, 2002.

[3] V. Grupta, G. A. Rincón-Mora, and P. Raha: Analysis and Design of Monolithic, High PSR, Linear Regulator for SoC Applications. Proceedings of the IEEE International SoC Conference: pp. 311-315, 2004.

[4] R. W. Erickson and D. Maksimovic: Fundamentals of Power Electronics. 2nd edition, Ed. Kluwer Academic Publishers, 2001.

[5] J. G. Kassakian, M. F. Schlecht, and G. C. Verghese: Principles of Power Electronics. Ed. Addison-Wesley, 1991.

[6] N. Mohan, T. M. Underland, and W. P. Robbins: Power Electronics: Converters, Applications and Design. Ed. John Wiley \& Sons, 1989.

[7] X. Zhou, P. L. Wong, P. Xu, F. C. Lee, and A. Q. Huang: Investigation of Candidate VRM Topologies for Future Microprocessors. IEEE Transactions on Power Electronics, vol. 15 ( $\mathrm{n}^{\mathrm{o}}$ 6): pp. 1172-1182, November 2000.

[8] B. Arbetter and D. Maksimovic: DC-DC Converter with Fast Transient Response and High Efficiency for Low-Voltage Microprocessor Loads. IEEE Applied Power Electronics Conference, pp. 156-162. 1998.

[9] G. Villar, E. Alarcón, F. Guinjoan, and A. Poveda: Efficiency-Oriented Switching Frequency Tuning for a Buck Switching Power Converter. IEEE International Symposium on Circuits and Systems (ISCAS'05): 2005. 